msh-mss Mathématiques et sciences humaines

155-156 | Automne-Hiver 2001

Varia

\title{
Genèse d'une théorie
}

Genesis of a theory

\section{Louis Frey}

\section{(2) OpenEdition}

\section{Journals}

Édition électronique

URL : http://journals.openedition.org/msh/2859

DOI : $10.4000 /$ msh.2859

ISSN : 1950-6821

\section{Éditeur}

Centre d'analyse et de mathématique sociales de l'EHESS

\section{Édition imprimée}

Date de publication : 1 décembre 2001

ISSN : 0987-6936

\section{Référence électronique}

Louis Frey, "Genèse d'une théorie », Mathématiques et sciences humaines [En ligne], 155-156 | Automne-Hiver 2001, mis en ligne le 10 février 2006, consulté le 23 juillet 2020. URL : http:// journals.openedition.org/msh/2859; DOI : https://doi.org/10.4000/msh.2859 
Math. \& Sci. hum. (39e année, n 156, 2001, p. 5-32)

\section{AVANT-PROPOS}

Louis Frey (1924-1999) fut longtemps (et jusqu'à sa retraite) titulaire de la chaire de Logique de l'Université de Provence (section de Philosophie).

Auteur d'une thèse mémorable sur une application des mathématiques à la critique textuelle ${ }^{1}$, il s'est surtout occupé, en étroite collaboration avec le Centre de mathématique sociale de l'École des hautes études en sciences sociales, de méthodes algébriques ou combinatoires pour la modélisation mathématique dans les sciences sociales $^{2}$

Les vingt dernières années de sa vie furent principalement consacrées à une recherche originale!: quelles propriétés des mathématiques connues par les contemporains de leur construction pourraient-elles éclairer, sinon expliquer, certaines proportions «!harmonieuses!» observées sur des édifices de l'Antiquité grecque et de l'Antiquité romaine!?

L'article publié ici est inédit. Louis Frey y montre comment et pourquoi il a été amené à élaborer une théorie purement conjecturale, comme il l'a lui-même souvent souligné, mais cohérente avec les mesures prises sur des monuments d'une part, les mathématiques de leur époque d'autre part.

C'est en hommage au scientifique et à l'ami que nous publions aujourd'hui un texte où il fournit l'essentiel de sa démarche.

M.B.

\footnotetext{
${ }^{1}$ Louis Frey, Analyse ordinale des évangiles synoptiques, La Haye et Paris, Mouton et Gauthier-Villars, 1972.

${ }^{2}$ Voir notamment!: Louis Frey, Techniques ordinales en analyse des données : algèbre et combinatoire, Paris, Hachette, 1971.
} 


\title{
GENÈSE D’UNE THÉORIE
}

\author{
Louis FREY
}

\begin{abstract}
RÉSUMÉ - Une théorie est présentée sur les représentations mathématiques et notamment arithmétiques, capables d'expliquer certaines des proportions observées dans des monuments des antiquités grecque et romaine.

MOTS-CLÉS - Approximations, Équation de Pell-Fermat, Médiétés, Partages d'un segment de droite, Architecture de l'Antiquité.
\end{abstract}

SUMMARY - Genesis of a theory

A mathematical (here, specifically arithmetical) theory is presented to explain many observed proportions in monuments of the ancient Greek and Roman civilizations.

KEYWORDS -Approximations, Pell-Fermat equation, Various means, Divisions of a line segment, Architecture of Antiquity.

Devant les membres du séminaire d'épistémologie de l'Université de Provence ${ }^{3}$, il m'a paru utile - et peut-être intéressant - de développer une sorte de réflexion critique sur ma propre évolution tout au long de dix années de recherche consacrées à l'architecture antique ou, plus précisément, à la manière dont les architectes antiques procédaient à l'élaboration du plan de leurs édifices. Comme vous le verrez, je suis en définitive parvenu à une théorie assez cohérente pour toute une classe d'édifices sur lesquels on retrouve les mêmes rapports exprimés par les mêmes couples de nombres. Mais ce ne fut pas sans tâtonnements, sans erreurs, sans fausses pistes abandonnées et parfois reprises jusqu'à ce qu'une meilleure interprétation ne finisse par les remplacer.

Tout d'abord, pourquoi cet intérêt apparemment saugrenu du logicien que je fus parmi vous, pour ce domaine incertain de l'architecture antique, domaine où ne cessent de fleurir les théories les plus contestables?

La raison profonde en fut un cours sur les quatre apories de Zenon relatives à l'impossibilité de rendre compte du mouvement d'une manière rigoureusement cohérente. Comme certains le soutiennent, ces apories concernent-elles effectivement, le problème du continu ou, comme d'autres le prétendent, le développement des mathématiques à la frontière des $\mathrm{VI}^{\mathrm{e}}$ et $\mathrm{V}^{\mathrm{e}}$ siècles ne permettait-il aucunement de même

\footnotetext{
${ }^{3}$ Séminaire d'épistémologie comparative de l'université de Provence et Institut de recherche sur l'architecture antique (I.R.A.A. - C.N.R.S.)
} 
formuler ce type de problème ? Il est toujours possible de se battre sur des textes ambigus et d'en proposer des interprétations divergentes alors que l'on ne dispose d'aucun traité systématique de la mathématique pré-euclidienne péniblement restituée à partir de fragments épars ou de témoignages tardifs.

La raison occasionnelle fut d'ordre simplement touristique : lors d'un séjour à Paestum et à Velia (l'ancienne Elée de Parménide et de Zénon) il m'apparût qu'il était possible en jouant avec des cailloux sur le sable de construire un discours apparemment cohérent pour rendre compte du nombre des colonnes du premier des temples de Paestum (dit «!la basilique!» ou «!Héraïon 1 !») et ce en recourant à la théorie pythagoricienne des nombres figurés. S'insinua alors l'hypothèse pernicieuse qu'à défaut de textes, les monuments pouvaient porter la trace des connaissances mathématiques de leur époque de construction d'où le titre d'un séminaire en décembre 1980 : «!Une arithmétique de pierres!» et l'élaboration d'un discours pour relier des nombres et leur donner un sens par l'intermédiaire d'un modèle de type arithmétique.

Faire parler de vieilles pierres est un rêve que bien d'autres ont caressé avec les excès que l'on sait sur la science secrète des pharaons, par exemple. Comment les interroger, qu'attendre d'elles ? Cela dépend évidemment de ce que l'on sait ou croit savoir et de ce que l'on présuppose. Force m'est aujourd'hui de reconnaître qu'à l'époque mes connaissances étaient assez rudimentaires et se limitaient aux nombres triangles, nombres carrés et autres nombres polygonaux dont nous parlent Nicomaque de Gérasa [30] et Théon de Smyrne [37]. Ils les attribuent explicitement aux premiers pythagoriciens lesquels, malheureusement, n'étaient sans doute pas encore installés à Crotone lors de la construction de ce premier Héraïon ! Certes, les chronologies sont incertaines, mais elles doivent inciter à la prudence.

Difficile cependant de résister à la tentation et de me confronter aussitôt à un autre des temples de Paestum, l'Athénaïon (ou encore «!temple de Céres!») entièrement «!publié!» par F. Krauss sur lequel je m'exerçais à une interprétation de type géométrique cette fois-ci à partir des valeurs des rapports entre les mesures des dimensions données par Krauss, rapports dont certains évoquaient la hauteur d'un triangle équilatéral, d'autres la diagonale d'un rectangle, etc. [26]. Les constructions géométriques évoquées par ces rapports (ou tracés régulateurs) furent exposées en premier lieu à un séminaire de Gilles Granger en avril 1981 puis à un séminaire de l'École pratique des hautes études en sciences sociales devant quelques archéologues parisiens. Leur réaction contre ces tracés régulateurs fut on ne peut plus vigoureuse et ma défense plutôt incertaine étant alors dans l'incapacité de répondre à la question fondamentale : Comment inscrire ces tracés dans la pierre avec des nombres ronds indispensables à l'entrepreneur ?. La plus belle volée de bois vert de ma vie de chercheur, ai-je souvent coutume de dire, amplement méritée et qui m'incita à des recherches plus approfondies et plus minutieuses au cours des trois années suivantes.

Ces nombres, je crus enfin les découvrir quelque temps plus tard sur un autre édifice, de Delphes cette fois-ci, le «!Trésor de Thèbes!», publié par J.-P. Michaud avec le maximum de précision dans l'estimation des dimensions et leur interprétation 
métrologique en unités de mesure antique [28]. Cet édifice ne pose aucun problème architectonique particulier car il s'agit d'un simple parallélépipède rectangle entourant deux pièces rectangulaires, le pronaos et la cella. En dépit de la simplicité de son plan comme de son élévation, cet édifice, construit par les Thébains pour commémorer leur victoire de Leuctres sur les Spartiates en - 371, est fort loin d'être rustique et ses proportions, d'après les divers archéologues qui l'ont étudié, témoigneraient d'une maîtrise architecturale parfaite.

Pour s'en tenir ici à l'essentiel, il se trouve que, pour l'élévation, la hauteur totale des murs depuis les orthostates jusqu'au larmier est égale, d'après J.-P. Michaud, à 275 dactyles, soit : $55 \square 5$. Cette dimension est, par ailleurs, dans des relations simples avec les autres dimensions de la façade, ce qui conduit à supposer qu'elle joue le rôle d'une grandeur de référence à partir de laquelle ont été déterminées les autres dimensions. Il en va de même pour les relations que cette dimension de 275 entretient avec les dimensions horizontales du pronaos et de la cella à condition de les prendre sur la ligne médiane des murs. En effet, la largeur commune du pronaos et de la cella est de 350 dactyles, la profondeur du pronaos en est exactement la moitié, soit 175, et la profondeur de la cella est de 445, soit : $89 \square 5$.

Coïncidence ou non, ces valeurs sont loin d'être quelconques. Notamment, grandeur de référence et profondeur de la cella sont dans le rapport de 275 à 445, soit : 55 à 89. Ces deux derniers nombres sont deux des termes de la suite dite de Fibonacci dont le rapport est une approximation de ce que l'on appelle aujourd'hui le «!nombre d'or!» et jadis, selon l'expression d'Euclide, le «!partage en extrême et moyenne raison!». Cette première constatation suggère un tracé régulateur directement dérivé de la construction de ce partage exposée par Euclide à la proposition 11 du deuxième livre des Eléments. Comme, par ailleurs, la poursuite de cette construction géométrique permet aussi d'obtenir la largeur commune de 350, qui est avec 275 dans le rapport de 14 à 11, on dispose ainsi d'un modèle géométrique montrant comment les trois dimensions importantes du plan sont coordonnées entre elles et dérivées de la grandeur de référence.

Ces considérations (ou élucubrations, penseront certains) ayant retenu l'attention des rédacteurs de la revue Mathématiques et Sciences Humaines, ont fait l'objet d'un article paru dans le numéro 88 de cette revue en 1984 [18bis]. Le lecteur curieux de vérifier le bien-fondé des notions exposées ci-dessus pourrait éventuellement s'y reporter mais l'intérêt principal de cette étude est moins dans ses résultats que dans ses manques et ses défauts et dont témoigne le titre même de l'article : «!le Trésor de Thèbes, deux modèles pour un monument!».

Deux modèles en effet, l'un de nature géométrique indiquant comment construire une certaine grandeur incommensurable avec telle autre, l'autre de nature arithmétique indiquant quels nombres entiers attribuer à ce couple de grandeurs pour les approximer de la meilleure façon possible. Ces modèles sont certes couplés sur cet édifice particulier mais ils n'en demeurent pas moins indépendants l'un de l'autre ce dont témoigne la conclusion : «!Ces analyses sont essentiellement descriptives. Elles 
retiennent certaines particularités du monument, elles les interprètent et les coordonnent dans un cadre théorique plausible, mais elles n'arrivent pas à déceler l'intention profonde du constructeur. Elles décrivent «!comment!» les choses ont été faites, elles ne découvrent pas «!pourquoi!» elles ont été faites ainsi!». Pourtant tous les éléments étaient là pour dire non seulement le «!comment!» mais aussi le «!pourquoi!», encore fallait-il pouvoir les coordonner et leur donner sens dans une théorie plus générale dont je ne disposais pas encore.

Divers facteurs sont intervenus dans la constitution progressive de cette théorie, parmi lesquels j'isolerais : un milieu de travail, un colloque sur l'approximation, une rencontre avec un mathématicien anglais ainsi qu'un facteur qu'il faut bien mentionner, la capacité de calcul d'un micro-ordinateur.

À partir de l'année universitaire 1984-85, le milieu de travail fut tout d'abord le séminaire de notre collègue Pierre Gros sur l'architecture antique bientôt élargi aux membres du bureau d'Aix de l'Institut de Recherche sur l'Architecture Antique (I.R.A.A.!- CNRS) devant lesquels il m'arrivait parfois de présenter mes interprétations d'un quelconque édifice grec ou romain. Auditoire amical, mais exigeant, qui ne se laissait pas facilement convaincre et qui m'incita à me situer uniquement dans le cadre de la mathématique antique d'où une étude plus poussée du langage des rapports et de l'ancienne théorie des proportions et des médiétés. Par ailleurs, de par leur localisation, les membres du bureau d'Aix étudient principalement des édifices romains et non pas des édifices grecs. Or il se révèle que l'architecture romaine est plus simple et plus systématique (plus monotone même) que l'architecture grecque. De ce fait, ses caractéristiques se dévoilent beaucoup plus aisément d'autant plus qu'il n'est en général guère d'ambiguïté sur l'unité de mesure utilisée, le pied romain standard (un pied = $0,2957 \mathrm{~m}$ ), et donc la valeur que les dimensions avaient pour le constructeur antique est connue avec certitude. Or ce sont presque toujours les mêmes nombres ou les mêmes couples de nombres qui réapparaissent sur les édifices les plus divers.

Ces couples de nombres sont précisément des approximations de ces grandeurs non-commensurables que sont la diagonale d'un carré ou d'un double carré avec le côté, donc de $\sqrt{2}$ ou de $\sqrt{5}$. C'est aux approximations que fut justement consacré un colloque du Centre d'Analyse et de Mathématique Sociale organisé en 1986 à Urbino en Italie (cf. [15]). Ce colloque me fut l'occasion de préciser une distinction pour moi désormais fondamentale entre : valeur approchée et approximation ${ }^{4}$.

Pour s'en tenir à la diagonale du carré, une valeur approchée de $\sqrt{2}$ est le résultat de la procédure d'extraction de la racine carrée, arrêtée à une certaine étape. Pour la plupart d'entre nous, elle est le nombre que nous indique une calculatrice de poche pris avec tant de décimales. Pour un archéologue, elle est un rapport de deux dimensions

\footnotetext{
${ }^{4}$ Louis Frey établit ici une distinction de sens, qui lui est commode pour la suite de son propos, entre les mots «!valeur approchée!» et «!approximation!». En mathématiques, ces deux termes sont usuellement synonymes (N.D.L.R.).
} 
dont la valeur se situe aux alentours de 1,4. D'une certaine manière, et par définition même de l'incommensurabilité, une valeur approchée n'est jamais une valeur exacte.

Corrélativement une approximation de $\sqrt{2}$ est constituée par un couple de nombres entiers dont l'un représente la longueur du côté et l'autre la valeur de la diagonale et ces deux nombres sont tels que le double du carré du premier diffère d'une unité en plus ou en moins du carré du second. Il en est ainsi du couple $(5,7)$ puisque : $5^{2} \square 2=50$ et $: 7^{2}=49$. Il en serait de même, comme le lecteur peut le vérifier, pour les couples $(12,17)$ et $(29,41)$. D'une certaine manière encore, une approximation est toujours exacte. De plus, elle est directement utilisable par un architecte qui dispose ainsi de «!nombres ronds!» lorsque, pour une raison ou une autre, il lui prend fantaisie de construire l'une des dimensions sur la diagonale du carré construit sur l'autre dimension 5 .

Mais ces approximations, qui se calculent d'une manière très systématique par la procédure dite d'anthyphérèse, quand donc furent-elles inventées ?

Les historiens de la mathématique antique en débattent et, parmi eux, un mathématicien anglais de l'Université de Warwick, David Fowler qui s'est pris de passion pour ce problème d'histoire des sciences au point d'y consacrer tout un ouvrage sérieusement documenté : «!The mathematics of Plato's academy!»[13]. Ayant la chance de le retrouver à chacune des vacances qu'il passe en France, je me suis familiarisé, grâce à lui, à la procédure du retranchement alterné décrite par Euclide sous le nom d'anthyphérèse et à l'implanter, sur ses indications, sur un micro-ordinateur. Il n'est désormais guère d'édifice qui ne passe à cette moulinette. Pour un monument romain, les résultats permettent de confirmer la valeur de l'unité de mesure et de retrouver à maintes reprises des couples de nombres du type $(5,7)$ et $(12,17)$ ou leurs multiples et sous-multiples. Pour un temple grec, où l'unité de mesure est toujours incertaine puisque chaque cité avait une valeur différente du pied, il n'est pas rare de retrouver par anthyphérèse sur les dimensions métriques ces couples d'approximations et ce, même si l'édifice est nettement antérieur à la période présumée de la découverte de l'anthyphérèse. L'architecture antique suppléerait-elle à l'absence de texte ? La question mérite à tout le moins d'être posée et étudiée avec soin.

Les archéologues spécialistes d'architecture antique aiment à présenter ce qu'ils appellent le «!tracé régulateur!» des édifices qu'ils étudient, tracé dans lequel interviennent des figures géométriques et des transformations effectuées sur elles : carrés, doubles carrés, rabattement de leurs diagonales, triangle équilatéral, parfois

\footnotetext{
${ }^{5}$ Ces couples sont des solutions (X, Y) en nombres entiers de l'équation (cf. § 2, infra)!: $2 X^{2} \sqcap Y^{2}=1$, d'où l'on déduit immédiatement!: $2=\frac{\square}{\square} \frac{\mathrm{Y}}{\square} \square^{2} \square+\frac{1}{\mathrm{Y}^{2}} \square$ i.e. $\sqrt{2}=\frac{\mathrm{Y}}{\mathrm{X}} \sqrt{1+\frac{1}{\mathrm{Y}^{2}}}$.

Le rapport exact (i.e. rationnel) $\frac{Y}{X}$ est donc une approximation par défaut de l'irrationnel $\sqrt{2}$. Cette approximation est d'autant meilleure que $\mathrm{Y}$ est grand (N.D.L.R.).
} 
pentagone, etc. Il s'agit en fait d'un modèle géométrique du monument, modèle qu'aurait utilisé son concepteur pour établir les principales proportions de son édifice. Il me devenait peu à peu évident que si de tels tracés avaient bel et bien été utilisés, pour déterminer la valeur des dimensions, l'architecte ne s'était pas livré à un calcul fastidieux de la valeur approchée mais avait directement utilisé, en consultant simplement une table, les approximations de ces couples de grandeurs théoriquement incommensurables.

Une première confirmation de cette hypothèse me fut apportée lors d'un séjour à Pompéi au cours de l'été 1987 en compagnie d'une équipe d'archéologues de l'université de Nimègue dirigée par notre collègue Jos De Waeme. L'un des participants, C. Peterse, qui mesurait l'une des grandes demeures pompéiennes, la Maison de Pansa [32], m'apporte un soir les valeurs en pieds osques de l'atrium de cette «!domus!» du -!II siècle. Grande fut ma surprise en constatant que les valeurs de toutes les dimensions étaient des nombres dérivés de la théorie des approximations. Ainsi l'une des pièces avait une largeur intérieure 12 pieds et une profondeur 17. La largeur intérieure de l'atrium était de 60 pieds et sa profondeur de 52, ce qui correspond à l'une des approximations de $\sqrt{3}$ par le couple $(15,26)$, et ainsi de suite, ce qui me permit d'établir, pratiquement sur le champ et sans aucun calcul de rapport, le tracé régulateur de cet atrium. L'expérience fut renouvelée sur trois autres atriums avec les mêmes résultats, seul un quatrième ne se pliait pas à ce type d'interprétation. Ce résultat m'amenait à penser qu'il n'y a modèle géométrique d'un édifice que si et seulement si il y a approximations.

Pour intéressant qu'il puisse être, un modèle géométrique n'en demeure pas moins uniquement descriptif. Il montre comment diverses grandeurs sont coordonnées entre elles et comment elles peuvent être dérivées d'une grandeur de référence mais il ne révèle pas la raison pour laquelle un architecte a choisi cette coordination particulière et non telle autre. Une esquisse de réponse me fut suggérée par le nombre d'édifices sur lesquels on décelait le recours à la diagonale du carré, ou à une partie de cette diagonale, correspondant à ce que l'on désignerait aujourd'hui par $\sqrt{2} \square 1$, obtenue par le rabattement du côté sur cette diagonale et enfin le rabattement de la partie restante sur le côté de manière à obtenir une segmentation du côté en deux parties de valeur : $\sqrt{2} \square 1$ et $2 \square \sqrt{2}$, (schéma décrit infra par la figure 2).

Que ce soit pour construire des rectangles ou pour segmenter une hauteur, dans quel but un architecte se livrerait-il à ce genre de construction géométrique ? À ma connaissance, aucun archéologue n'apporte le moindre élément de réponse. Il faut se retourner vers les historiens de la mathématique grecque pour découvrir qu'il s'agit de ce que l'on appelle le partage harmonique d'une droite (cf. $\S 5$, infra).

- Partage harmonique donc sur certains monuments tels, par exemple, la Porte Noire de Besançon!;

- Partage géométrique aussi sur d'autres tel le Trésor de Thèbes cité plus haut!; 
- Partage arithmétique aussi lorsque l'un des segments est le double d'un autre comme à l'Athénaïon de Paestum où la hauteur de la colonne est exactement le double de la hauteur de l'entablement.

Ces trois partages conduisent inéluctablement vers la très antique et très vénérable théorie des médiétés. Pour envisager cette interprétation, pour songer à cet emprunt des mathématiques, ou à cette retombée utilitaire, encore fallait-il avoir étudié suffisamment d'édifices pour relever la constance de ces partages, parfois tous les trois associés sur un même édifice, tel que l'arc de triomphe d'Orange dont les proportions sont tellement bien agencées qu'il constitue pour moi une véritable leçon d'architecture romaine.

Ainsi, d'après l'idée commune (mais peut-être pas totalement fausse pour certains grands architectes antiques) selon laquelle l'architecte est le «!maître des proportions!», l'élaboration d'un plan et l'établissement des rapports entre les diverses dimensions se seraient effectués en prenant pour modèle la théorie mathématique des proportions. Telle est du moins l'hypothèse qui commençait à prendre corps et que ne cessaient de confirmer les divers édifices qu'il m'arrivait d'étudier, et notamment des édifices romains de toute nature : temples évidemment, assises d'autel, mais aussi demeures privées, amphithéâtres, arcs de triomphe, plans de forums, remparts de ville et jusqu'à des centres commerciaux. Il serait abusif d'en déduire que tout architecte romain était aussi géomètre. Beaucoup plus simplement, des canevas ou des normes avaient été établis et répandus dans tout l'empire au point que la grande porte d'entrée du péristyle du temple de Bel à Palmyre présente exactement les mêmes proportions que la porte du pronaos de la Maison Carrée de Nîmes.

Canevas et normes présupposent des documents et des textes dont aucune trace ne nous serait parvenue. Est-ce totalement exact ? N'existe-t-il vraiment aucun texte ou ne sait-on plus interpréter ceux qui subsistent et notamment le seul traité d'architecture que nous ait légué l'Antiquité, à savoir le «!De Architectura!» de Vitruve, dédié par son auteur à Auguste?

Au cours du mois de septembre 1989 j'ai eu la chance de disposer du manuscrit de la traduction du livre III de ce traité, traduction effectuée avec toute la rigueur souhaitable par notre collègue Pierre Gros pour le compte des éditions G. Budé [19]. Vitruve consacre ce troisième livre à la seule description des temples ioniques répartis en cinq types qui sont principalement caractérisés par la relation entre l'espacement des colonnes et leur hauteur.

Étudiant alors les nombres que donne l'auteur en termes de module (le diamètre de la colonne) j'ai eu la très grande surprise, et aussi la satisfaction, de constater que pour deux de ces types, à savoir le diastyle et l'eustyle ${ }^{6}$ qui semblaient avoir la préférence de Vitruve, ces nombres étaient exactement ceux des approximations des partages harmoniques et géométriques. Ces nombres se retrouvaient et dans le rythme de la

\footnotetext{
${ }^{6}$ Diastyle!: édifice dont les colonnes sont à distance l'une de l'autre égale à trois fois leur diamètre. Eustyle!: même chose, mais avec deux diamètres 1/4 d'espacement (N.D.L.R.)
} 
colonnade, et dans la découpe du chapiteau ionique, et enfin dans la découpe des deux entablements possibles dans l'ordre ionique. Pratiquement donc, toutes les dimensions du diastyle et de l'eustyle relevaient de la théorie des médiétés et de la théorie des approximations des grandeurs incommensurables et la concordance était rigoureusement parfaite entre les nombres prévus par le modèle et les nombres donnés par Vitruve.

Cette interprétation a reçu l'assentiment du traducteur et a fait l'objet d'un article détaillé qui vient de paraître dans la Revue Archéologique à laquelle le lecteur soucieux de détail pourra se reporter [18]. Des conclusions déduites des nombres du $D e$ Architectura, et bien que Vitruve ne mentionne nulle part ni les médiétés ni leurs approximations, il résulte que ce livre III constitue, à mes yeux, le témoignage textuel certain du recours, de la part des architectes antiques, à des théories élaborées par des mathématiciens pour définir des proportions et donner leur traduction en nombres entiers.

Pour résumer, on a d'une part des édifices, en assez grand nombre, qui suggèrent finalement une certaine théorie suggérant une raison à tout le moins plausible aux choix effectués dans l'établissement des dimensions, d'autre part, un texte dans lequel on retrouve les mêmes types de déterminations venant confirmer les hypothèses déduites des données empiriques. Ainsi, au terme de cette recherche, la théorie des approximations des grandeurs incommensurables répond à la question initiale : comment traduire en nombres ronds un tracé régulateur? De plus, une raison semble aussi avoir été trouvée au choix de tel tracé particulier d'un édifice donné. Non pas pour tous les édifices antiques, il importe de le souligner, mais pour toute une classe d'entre eux, la théorie des médiétés paraît effectivement constituer le cadre d'interprétation qui donne un sens au choix de tel et tel rapport relevés entre les dimensions. Jusqu'ici, ce cadre n'a que très sommairement été évoqué et il convient de l'exposer d'une manière plus détaillée d'autant que les principales notions sur lesquelles il repose sont quasiment oubliées de tous aujourd'hui à l'exception de quelques spécialistes de l'histoire des mathématiques.

\section{LE LANGAGE DES RAPPORTS}

Pour autant qu'un architecte poursuit une visée esthétique, il détermine ses dimensions en fonction de rapports privilégiés à ses yeux et il les coordonne dans des systèmes de proportions considérées par lui comme harmonieuses. À qui souhaite découvrir les grandes lignes de son projet, il importe de déceler tout d'abord quels sont les rapports et les proportions mis en œuvre et il ne saurait y parvenir sans recourir à la manière qui avait alors cours de concevoir et de traiter ces rapports et ces proportions [22] et [29].

Un rapport est une relation sur un couple de grandeurs homogènes et, dans l'Antiquité cette relation se décrit en indiquant comment l'une des grandeurs se construit à partir de l'autre. Prenons pour exemple la relation entre les largeurs du triglyphe et de 


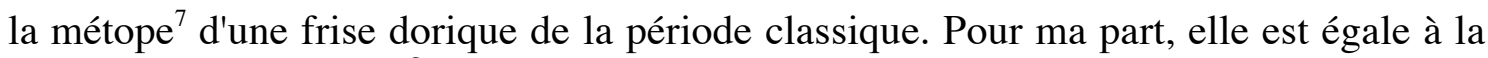
fraction irréductible : $\frac{2}{3}$. Ce n'est absolument pas le langage des anciens. Grecs et Romains ont une conception opératoire du rapport. Ils considèrent que la largeur de la métope se construit à partir de celle du triglyphe par l'adjonction de sa moitié, soit : $1+\frac{1}{2}$, ou, symétriquement, que le triglyphe dérive de la métope par le retrait de son tiers, soit : $1 \square \frac{1}{3}$. D'une certaine manière, il est supposé qu'une transformation est effectuée sur l'une des grandeurs pour obtenir l'autre et un grand nombre de ces transformations reçoivent un nom qui les caractérise et permet de définir une classe de couples, qui sont tous tenus pour différents, mais qui ont une propriété commune : «!être égal à l'autre plus sa moitié!» dans l'exemple choisi. Il se construit ainsi un véritable lexique qu'il serait trop long d'exposer ici mais qu'il est néanmoins utile de connaître car il y a tout lieu de penser que les architectes y recouraient pour déterminer des couples de dimensions (cf. [22] et [29]).

Il est donc souhaitable de pouvoir retrouver ces transformations d'une grandeur en une autre. Toutefois, sauf pour les valeurs simples, les rapports calculés sur les mesures métriques ne les mettent pas directement en évidence. Comment, par exemple, interpréter un rapport égal à 1,1428571 ? Qui, du premier coup d'œil y lira la transformation : $1+\frac{1}{7}$ ? (relation dénommée : sesquiseptime ou ephebdomos). Il est alors fort utile de disposer d'un algorithme qui réduise à des nombres entiers simples nos relevés métriques. Cet algorithme existe depuis fort longtemps, et il n'est autre que l'anthyphérèse euclidienne déjà évoquée plus haut.

À ma connaissance, le mathématicien David Fowler est le premier à avoir proposé aux archéologues le recours à l'anthyphérèse lors d'un débat avec l'archéologue anglais J.J. Coulton, débat résumé dans un article non encore publié et au titre fort évocateur : «!An objective and practical method for describing and understanding ratios!». Méthode objective et fort simple qui consiste à retrancher le plus petit de deux termes autant de fois que de besoin, puis à renouveler le même retranchement avec le reste obtenu et ainsi de suite jusqu'à l'obtention d'un dernier reste suffisamment petit pour que l'on décide de le négliger. Arrêtant alors les retranchements, on égalise les deux derniers termes et, remontant chacune des étapes précédentes, on exprime chacun de leurs termes en fonction du dernier. Cette procédure est très aisément programmable. Comme je l'indiquais précédemment j'utilise systématiquement cet algorithme qui donne des réductions en nombres entiers (describing). Assez fréquemment, celles-ci diffèrent de celles proposées par les archéologues et se révèlent plus précises, mais surtout plus significatives lorsqu'elles aboutissent à ces nombres particuliers dérivés de la théorie des approximations (understanding).

\footnotetext{
${ }^{7}$ Métope!: intervalle séparant deux triglyphes. Triglyphe!: ornement de la frise dorique comportant deux glyphes et deux demi-glyphes. Glyphe!: trait gravé en creux dans la pierre (N.D.L.R.)
} 


\section{LES APPROXIMATIONS}

Depuis qu'elles sont évoquées, il importe de définir avec précision ce que l'on appelle l'approximation de la racine carrée d'un entier $k$ (qui n'est pas un carré parfait). D'une manière générale on convient d'appeler «! approximation d'ordre 1 !» tout couple de deux nombres entiers $\mathrm{X}$ et $\mathrm{Y}$ tels que $k$ fois le carré du premier soit égal à une unité près au carré du second. Cette définition s'exprime par l'équation suivante, souvent appelée par les mathématiciens contemporains : «!équation de Pell-Fermat!» :

Couple $(\mathrm{X}, \mathrm{Y})$ d'entiers vérifiant : $\left(k \mathrm{X}^{2}\right)-\mathrm{Y}^{2}= \pm 1(k \square \mathrm{N})$

Lorsque deux entiers vérifient cette équation, on convient de poser $: \mathrm{X} \sqrt{k} \approx \mathrm{Y}$

Certaines de telles approximations de $\sqrt{2}$ ont déjà été évoquées avec les couples!: $(5,17)$ et $(12,17)$ ou encore $(29,41)$. On posera donc : $5 \sqrt{2} \approx 7$, ou : $12 \sqrt{2}: 17$ et l'on acceptera de dire que 7 est la diagonale (rationnelle) de 5 ou 17 la diagonale de 12.

Parmi les approximations de $\sqrt{5}$, on mentionnera le couple $(4,9)$ pour lequel : $4^{2} \square 5=80$ et $9^{2}=81$ et le couple $(17,38)$ qui présente la même caractéristique. On convient de poser : $4 \sqrt{5} \approx 9$ ou : $17 \sqrt{5} \approx 38$ et de dire que 38 est la diagonale d'un double carré de côtés 17 et 34 .

Parmi les approximations de $\sqrt{3}$, signalons les couples $(4,7)$ et $(15,26)$ avec : $15^{2} \square 3=675$ et $26^{2}=676$. On posera : $15 \sqrt{3} \approx 26$ et la hauteur d'un triangle équilatéral de côté 30 sera considérée comme égale à 26 .

\subsection{APPROXIMATIONS DÉRIVÉES}

On dispose ainsi de couples de nombres qui permettent d'approximer l'inexprimable, mais ce ne sont pas les seuls que l'on rencontre en architecture antique. De fait, si un architecte a recours aux mathématiques, il n'en est pas pour autant l'esclave. Ses dimensions peuvent avoir des valeurs quelconques qui ne coïncident pas forcément avec les couples de nombres que le mathématicien lui fournit et la liste des approximations d'ordre 1 constitue un lexique trop restreint pour ce qu'il doit réaliser. Il lui est donc indispensable d'étendre ce lexique et de recourir à des approximations dérivées, c'est-àdire à des couples dont les différences sur les carrés, tout en restant petites, seront supérieures à l'unité. Il se trouve heureusement des manières systématiques de procéder à l'extension du glossaire initial sans en atténuer la rigueur. Parmi les extensions rencontrées on relève notamment l'application de règles telles que :

Un carré ou un double carré de côté double auront une diagonale double.

Si 17 est diagonale de 12, 34 sera admis comme diagonale de 24 .

On vérifie alors que $24^{2} \square 2=1152$ et $34^{2}=1156$, la différence est maintenant de deux unités. 
De même pour un double carré de côtés 17 et 34 , si 38 est diagonale de 17, 76 sera diagonale de 34 , avec : $34^{2} \square 5=5780$ et $76^{2}=5776$.

Que ces couples de nombres se rencontrent en architecture antique, cela est indéniable et, comme la «!Maison de Pansa!» en est un exemple parmi bien d'autres, il arrive aux archéologues de les rencontrer mais, évidemment, sans percevoir qu'il s'agit d'approximations. Ils ne sont d'ailleurs absolument pas responsables de cette lacune puisque la théorie des approximations (ou des fractions continues, dans un langage plus moderne) est tombée en désuétude et ne se trouve enseignée nulle part.

Il est cependant à signaler que ces nombres se retrouvent dans un texte du XIII siècle [33] qui conseille : «!Si tu veux faire croître exactement le carré, allonge la lisière $\mathrm{du}$ quart et du sixte et cette dernière est la ligne diagonale autant qu'on peut l'approcher!» ce qui correspond au couple $(12,17)$ puisque : $12 !+! 3 !+! 2 !=! 17$. Par ailleurs, dans son traité d'architecture, Philibert de l'Orme au XVI ${ }^{\mathrm{e}}$ siècle justifie le recours à l'approximation dérivée $(7,10)$ et utilise les approximations $(5,7)$ et $(12,17)$. Le recours à des approximations en architecture s'est donc poursuivi au moins jusqu'au $\mathrm{XVI}^{\mathrm{e}}$ siècle avant de sombrer dans les oubliettes de l'histoire (cf. [8]).

Toutefois la question fondamentale à nos yeux est : «!pourquoi ces couples d'entiers ?!» ou la question corrélative : «!pourquoi vouloir déterminer les dimensions d'un édifice à partir de grandeurs incommensurables ?!» et nos architectes contemporains seraient bien en peine d'y répondre. L'une des réponses plausibles réside dans le désir de ces anciens constructeurs de réaliser entre les dimensions de leurs monuments tout un jeu de proportions qui, aujourd'hui, ne sont connues que de quelques spécialistes de la mathématique grecque lesquels, en général, ne s'intéressent guère à l'architecture.

Aujourd'hui, si l'on parle de proportion entre trois termes, il n'en est qu'une seule, que les Anciens appelaient «!la proportion continue!», définie par l'égalité d'un rapport : $\frac{a}{b}=\frac{b}{c}$. Dans l'Antiquité, cette proportion était certes connue et utilisée mais elle faisait partie d'un système plus large regroupant d'autres relations aussi définies sur trois termes à partir non pas d'un rapport direct entre les termes mais d'un rapport entre les différences des termes. Pour éviter toute ambiguïté, on conviendra de parler en ce cas de médiété et la proportion continue n'était que l'une des médiétés parmi d'autres définies à l'époque d'Euclide. Cette notion de médiété, qui fut l'un des sommets de l'arithmétique néo-pythagoricienne, est tout aussi ignorée de nos jours que la théorie des approximations et il n'est donc pas inutile d'en exposer brièvement les éléments.

\section{LES MÉDIÉTÉS NUMÉRIQUES}

Fondamentalement, une médiété est un triplet de nombres vérifiant une relation donnée. Je retiendrai les quatre médiétés qui se rencontrent en architecture, définies sur trois termes : $a$ étant le «!grand!», $c$ étant le «!petit!» et $b$ étant le «!moyen!» [30] et [31]. 
a) Médiété arithmétique

Le moyen diffère du petit et du grand de la même part de lui-même, soit encore :

avec $: b=\frac{(a+c)}{2} b$

$$
\frac{a \square b}{b \square c}=\frac{a}{a}
$$

b) Médiété géométrique ( ou proportion)

Chaque terme excède l'autre de la même part, soit encore :

$$
\frac{a \square b}{b \square c}=\frac{a}{b}
$$

avec $: b=\sqrt{a c}$

Exemple : $(1,2,4)$

c) Médiété harmonique

Les deux extrêmes sont dans une relation inversée avec le moyen, soit encore!:

$$
\frac{a \square b}{b \square c}=\frac{a}{c}
$$

avec $: \quad b=\frac{2 a c}{a+c}$ 吕.e. $\frac{1}{b}=\frac{1}{2} \frac{1}{\square}+\frac{1}{c}+\square \quad$ Exemple $:(2,3,6)$

d) Médiété sous-contraire à la géométrique

Directement définie par la solution b) positive de :

$$
\frac{a \square b}{b \square c}=\frac{b}{a}
$$

Exemple : $(1,4,6)$

\section{LES MÉDIÉTÉS DE PARTITION}

Définie d'abord en arithmétique, la théorie des médiétés à son correspondant en géométrie avec les médiétés dites de partition d'un segment de droite. Il s'agit, cette fois-ci, non pas de trouver trois entiers qui vérifient telle ou telle relation mais de partager un segment de droite $\mathrm{AB}$ en un point $\mathrm{C}$ de telle sorte que les segments $\mathrm{AC}$, $\mathrm{BC}$ et le segment entier $\mathrm{AB}$ vérifient les rapports de l'une des quatre médiétés. On parle alors de «!partage arithmétique, ou géométrique, ou harmonique!».

En raison des contraintes qu'impose le partage d'une droite, les valeurs des moyens correspondants sont maintenant complètement déterminées car elles deviennent les solutions d'équations du premier ou du second degré et l'on aura :

\subsection{PARTAGE ARITHMÉTIQUE D'UNE DROITE}

Prenons l'exemple de l'Athénaïon de Paestum pour lequel la hauteur de la colonne est le double de la hauteur de l'entablement [26]. On peut représenter les segments de ce 
partage par les nombres : colonne $=2$, entablement $=1$, hauteur totale $=3$. La hauteur de la colonne est la moitié de la somme : entablement + hauteur totale dont elle constitue la moyenne arithmétique. D'une manière générale, dès qu'une dimension est divisée en deux segments dont l'un est le double de l'autre on est en droit de parler de médiété arithmétique de partition et le rapport du moyen $b$ au grand terme $a$ est égal à!: $\frac{b}{a}=\frac{2}{3}$.

\subsection{PARTAGE GÉOMÉTRIQUE D'UNE DROITE}

Couper une droite de telle sorte que les segments vérifient le rapport de la médiété géométrique revient à déterminer deux nombres $a$ et $b$ tels que $b^{2}=a(a-b)$, d'où!: $\frac{\square}{\square} \square_{a}^{2}+\frac{\square}{\square} \square \square \square 1=0$. C'est donc résoudre l'équation du second degré : $x^{2}+x-1=0$ dont la racine positive est : $\frac{b}{a}=\frac{(\sqrt{5} \square 1)}{2}=0,61803 \ldots$

Son inverse, égal à : $\frac{(\sqrt{5}+1)}{2}=1,61803 \ldots$ est aujourd'hui improprement appelé le «! nombre d'or!». Ce nombre est fréquemment représenté par le symbole : «! $\varnothing$ !» pour lequel $\frac{1}{\varnothing}=\varnothing \square 1$ et : $\varnothing^{2}=\varnothing+1$.

Depuis la Renaissance italienne, qui a retenu cette seule médiété sous le nom plus exact de «!divina proportione!» (Luca Pacioli) ou de «!sectio aurea!» (Léonard de Vinci), le nombre d'or jouit d'une célébrité excessive et certains, qui ne connaissent que lui, prétendent le retrouver en des lieux où il n'a véritablement que faire alors que d'autres plaquent des pentagones simples ou étoilés sur n'importe quel monument. Ces abus ne doivent cependant pas nous interdire de le retrouver lorsqu'il est effectivement utilisé comme c'est le cas pour le rythme de la colonnade de la façade d'un temple eustyle à six colonnes où l'on retrouve sur les entre-axes les approximations de cette «!sectio aurea!» par les nombres de Fibonacci.

\subsection{PARTAGE HARMONIQUE D'UNE DROITE}

Bien moins connu, on peut même dire totalement ignoré de nos jours, est le partage harmonique d'une droite. Couper une droite de telle sorte que les segments vérifient les rapports de la médiété harmonique revient à déterminer $a$ et $b$ tels que!:

$(a-b)^{2}=a(2 b-a)$, d'où !: $\frac{\square b}{\square a} \square^{2} \square 4 \frac{\square}{\square} \square \square$ $x^{2}-4 x+2=0 \quad$ dont la racine supérieure à l'unité est égale à : $\frac{b}{a}=2 \square \sqrt{2}=0,58578 \ldots$

Cette valeur a pour complément à l'unité : $\sqrt{2} \square 1$ d'où la construction évoquée plus haut du partage harmonique par le rabattement d'un côté sur la diagonale (cf. infra figure 2). 


\subsection{PARTAGE SOUS-HARMONIQUE D'UNE DROITE}

C'est le nom que j'ai choisi de donner à la découpe d'une droite selon la quatrième de nos médiétés dont l'équation $a(a-b)=(2 b-a) b$, d'où $2 \frac{\square^{b}}{\square} \square_{a}^{2}=1$ a pour solution!: $\frac{b}{a}=\frac{1}{\sqrt{2}}=0,70701 \ldots$

Ces deux derniers partages, harmonique et sous-harmonique, sont très proches l'un de l'autre puisqu'ils se définissent et se construisent géométriquement à partir de la diagonale d'un carré alors que le partage géométrique se définit et se construit à partir de la diagonale d'un double carré. Il peut, certes, se trouver qu'ils soient simultanément utilisés sur le même édifice, et l'arc de triomphe d'Orange en est un exemple, mais en général il semblerait qu'un monument privilégie soit le carré, avec ses deux partages, soit le double carré avec son partage «!doré!». Ainsi, dans le traité de Vitruve, la colonnade d'un temple ionique diastyle ne fait intervenir que les partages harmoniques et sous-harmonique alors que celle d'un temple eustyle ne fait intervenir que le partage géométrique. De même est-il curieux de relever que dans son traité d'architecture de 1567 Philibert De l'Orme ne mentionne que des constructions à partir de rabattements de diagonales de carrés et passe complètement sous silence la «!sectio aurea!» alors que la «!divina proportione!» de Luca Pacioli est parue en 1509.

La médiété harmonique, qui aurait reçu son nom d'Archytas de Tarente (cf. Ivor Thomas [25], p. 113), évoque une interprétation musicale, d'autant plus que ce qualificatif est justifié en ces termes par Nicomaque de Gerasa : «!Les sommets de tout cube sont 12 , ses angles sont 8 , ses plans sont 6.8 est donc la médiété harmonique de 6 et de 12. La quarte est de 8 à 6 puisque épitrite, la quinte est de 12 à 8 puisque hémiole, l'octave, qui est l'union des deux, est de 12 à 6 puisque double. C'est donc d'une façon tout à fait appropriée que cette médiété a été appelée harmonique!» [30].

À en croire Paul Tannery il en irait de même du partage sous-harmonique. Ce spécialiste de la mathématique hellénique écrit en effet en 1902 à propos du rôle de la musique grecque dans le développement de la mathématique pure : «!Il ne restait qu'à aborder la question mathématiquement, et tout d'abord, savoir si l'octave avait une moitié. ... On ne saurait nier le rôle joué dès lors par le problème musical, et il dût au moins amener de nouvelles recherches pour une approximation aussi exacte que possible de $\sqrt{2}$ !» [35]. Ces types de proportions mathématiquement exactes, musicalement agréables, ont pu tenter les architectes antiques pour les aider à construire des monuments parfaitement équilibrés.

\section{CONSTRUCTIONS GÉOMÉTRIQUES ET APPROXIMATIONS}

Les constructions des trois partages, géométrique, harmonique et sous-harmoniques résultent directement de leur définition. Elles sont très simples, rudimentaires même, et 
l'on peut les considérer comme les constructions élémentaires qui interviennent dans l'élaboration du tracé régulateur d'un édifice. Un tel tracé associe plusieurs de ces constructions, souvent enchaînées les unes aux autres et développées à partir de la grandeur de référence qui, assez généralement, est soit la hauteur de la colonne, soit une distance entre des axes de colonne.

\subsection{LE PARTAGE GÉOMÉTRIQUE (cf. figure 1 en annexe)}

On sait que, en vertu du théorème de Pythagore, la diagonale d'un double carré de côtés 1 et 2 , a pour valeur $\sqrt{5}$. La moitié de cette diagonale vaut donc : $\frac{\sqrt{5}}{2}$.

Si on lui rajoute le demi petit côté, on obtient : $\frac{\sqrt{5}+1}{2}=\varnothing$.

Si on le lui retranche, on obtient : $\frac{\sqrt{5} \square 1}{2}=\frac{1}{\varnothing}$.

Un tel partage rend compte du placement des colonnes des temples ioniques de type eustyle décrit par Vitruve. Je l'ai aussi retrouvé dans la découpe d'une élévation, celle de l'arc de triomphe d'Orange où les différents segments correspondent à la hauteur du piédestal, à celle de la colonne et à celle du premier attique. Une découpe en tout point analogue se retrouve sur le temple de Segeste, antérieur à l'arc d'Orange de près de cinq siècles.

Cette construction peut aussi être utilisée pour obtenir des rectangles «!dorés!» [5]. De tels rectangles paraissent avoir été utilisés pour le placement des colonnes des propylées de l'acropole d'Athènes, non pas sur les axes des colonnes, mais sur les rectangles d'inscription des colonnes.

Les approximations en nombres entiers du partage géométrique dérivent des approximations de $\sqrt{5}$ au moyen de l'équation $5 x^{2}-y^{2}= \pm 1$.

Si, par exemple, on part de l'approximation : $4 \sqrt{5} \approx 9$ et d'un double carré de côtés 8 et 16 , on aura : $\frac{8(\sqrt{5}+1)}{2}=9+4=13$ et $\frac{8(\sqrt{5} \square 1)}{2}=9-4=5$. On posera donc $: 8 \square \varnothing \approx 13$ et $8 \square \varnothing \approx 5$.

De même, à partir de l'approximation $17 \sqrt{5} \approx 38$, pour un double carré de côtés 34 et 68 , on obtiendrait : $34 \square \varnothing \approx 55$ et $\frac{34}{\varnothing} \square 21$.

On constate que l'on obtient ainsi une suite de nombres : 5, 8, 13, 21, 34, 55, qui sont tels que, à partir du troisième, chaque nombre est la somme des deux précédents et que le rapport de deux nombres consécutifs est une approximation de $\varnothing$. Cette suite, que l'on peut compléter indéfiniment, est aujourd'hui connue sous le nom de : «!Suite de Fibonacci!». Elle est évidemment bien antérieure à ce mathématicien du XIII ${ }^{\mathrm{e}}$ siècle qui l'a peut-être recueillie lors d'un séjour à Cordoue et qui l'avait proposée dans une 
perspective toute différente. Il se trouve (est-ce dû au seul hasard ?) que si l'on exprime les hauteurs de l'arc d'Orange en nombre de rayons de colonne, les valeurs obtenues sont exactement celles de cette suite. Il en va de même, comme je l'ai mentionné ci-dessus, pour les distances axiales indiquées par Vitruve pour les temples ioniques eustyles où elles interviennent par leur moitié : $4 ; 6,5 ; 10,5$ et 17 (cf. [18]).

\subsection{LE PARTAGE HARMONIQUE (cf. figure 2 en annexe)}

Nul ne mentionne ni, à plus forte raison, n'utilise le partage harmonique qui pourtant, à mon expérience du moins, paraît avoir été largement utilisé surtout dans l'architecture romaine.

Il convient donc de diviser une droite en deux segments de valeur complémentaire!: $2 \square \sqrt{2}$ et $\sqrt{2} \square 1$. L'un des moyens les plus simples est de rabattre un côté du carré sur la diagonale et de rabattre le segment ainsi délimité, de valeur : $\sqrt{2} \square 1$, sur le côté opposé. Un autre moyen pour obtenir deux segments qui soient dans le même rapport harmonique consiste à rabattre la demi-diagonale sur le prolongement du côté. Ces deux segments vérifient : $(\sqrt{2} \square 1) \square \sqrt{2}=2 \square \sqrt{2}$.

Pour obtenir les valeurs entières d'un partage harmonique, on retiendra les trois premiers couples des approximations d'ordre 1 de $\sqrt{2}:(5,7) ;(12,17) ;(29, ! 41)$ et les trois couples d'approximations dérivées : $(7,10) ;(17,24) ;(41,58)$. Leur présentation a été faite sous forme de tableau (cf. page suivante).

Ces nombres se retrouvent sur maints édifices romains. Ainsi la porte d'entrée du pronaos de la Maison Carré de Nîmes est inscrite dans un rectangle harmonique comme la grande entrée du temple de Bel à Palmyre. Pour s'en tenir à un texte, d'après Vitruve le rectangle d'encadrement de deux colonnes consécutives des temples diastyle est un rectangle harmonique de hauteur égale à 8 rayons et demi et de largeur égale à 5 rayons, soit le couple $(17,10)$.

\begin{tabular}{cccccc}
\hline$\sqrt{2}$ & Valeur approchée & $2 \square \sqrt{2}$ & Valeur approchée & $\sqrt{2} \square 1$ & Valeur approchée \\
\hline $5 \sqrt{2} \approx 7$ & 1,4 & $(5,3)$ & 0,6 & $(5,2)$ & 0,4 \\
\hline $7 \sqrt{2} \approx 10$ & 1,428 & $(7,4)$ & 0,571 & $(7,3)$ & 0,428 \\
\hline $12 \sqrt{2} \approx 17$ & 1,4166 & $(12,7)$ & 0,5833 & $(12,5)$ & 0,4166 \\
\hline $17 \sqrt{2} \approx 24$ & 1,4117 & $(17,10)$ & 0,5882 & $(17,10)$ & 0,4117 \\
\hline $29 \sqrt{2} \approx 41$ & 1,4138 & $(29,17)$ & 0,5862 & $(29,12)$ & 0,4138 \\
\hline $41 \sqrt{2} \approx 58$ & 1,4146 & $(41,24)$ & 0,5853 & $(41,17)$ & 0,4146 \\
\hline
\end{tabular}




\subsection{LE PARTAGE SOUS-HARMONIQUE (cf. figure 3 en annexe)}

Rien de plus simple que d'obtenir ce partage. Il suffit de rabattre sur l'un des côtés la demi-diagonale d'un carré, ou encore de rabattre la diagonale entière sur le prolongement d'un côté. Quant à ses valeurs entières, elles figurent déjà sur le tableau précédent puisqu'elles ne sont autres que les approximations de $\sqrt{2}$.

Il arrive que les archéologues mentionnent dans leurs tracés régulateurs des rabattements de diagonale du carré. Mais ils n'interprètent évidemment pas cette construction en terme de médiété et n'aperçoivent pas qu'elle est très souvent utilisée en même temps que celle d'un partage harmonique. Ils ne se soucient guère, non plus, d'indiquer quelles valeurs entières, en unités antiques, correspondent à cette construction et ils se contentent d'indiquer la valeur du rapport sur les relevés métriques. Il leur suffit que cette valeur soit comprise entre 1,4 et 1,42 pour proposer ce type de rabattement qu'ils supposent souvent avoir été effectué au compas ou au cordeau.

Pour ma part, ne serait-ce que pour l'établissement du projet soumis aux autorités et l'établissement du devis initial, j'ai tendance à penser qu'il n'en est rien et que les dimensions ont été déterminées avant toute mise en œuvre sur le chantier. J'adopte donc une hypothèse assez restrictive qui n'admet de tels modèles géométriques par rabattement de diagonales de carrés ou de doubles carrés que s'ils se trouvent associés à des approximations. Que certains architectes partagent ce point de vue, j'en prendrai pour témoin Philibert de l'Orme qui se méfie du compas au point de déclarer en justification de l'approximation de $\sqrt{2}$ par le couple $(7,10)$ :

\footnotetext{
Si est-ce que pour le mesurer avecques le compas, il ne se peut faire si iustement, qu'il ne s'en faille quelque peu, combien que la pointe dudit compas soit fort subtile, car elle en emporte touiours quelque quantité, qui est cause que le rapport ne se peut trouver iustement (Livre II, folio 38)
}

Un des exemples de ce remplacement du compas par les nombres est donné par la découpe des diverses hauteurs du chapiteau toscan proposée par Philibert de l'Orme au folio 140. L'auteur ne mentionne pas la raison de ce découpage. Il indique simplement les hauteurs de chaque partie en termes de module, égal à la douzième partie du rayon de la colonne. Comme par hasard, ces nombres correspondent exactement aux partages harmoniques et sous-harmoniques construits sur des carrés de 12 et de 7 comme on peut le constater sur la figure 4 (cf. en annexe).

$$
\begin{array}{ll}
\text { Pour : } & R=12, \\
& R(\sqrt{2} \square 1)=17-12=5=\mathrm{E}+\mathrm{F} \\
& \text { et }: 5(\sqrt{2} \square 1)=7-5=2=\mathrm{L} \\
& \mathrm{R}(2 \square \sqrt{2})=12-5=7=\mathrm{K}+\mathrm{I}+\mathrm{H} \\
& 7(\sqrt{2} \square 1)=10-7=3=\mathrm{H} \\
& \text { et }: 7 \square \frac{7}{\sqrt{2}}=7-5=2=\mathrm{K}
\end{array}
$$


Cette analyse des proportions du chapiteau toscan est reportée sur la figure 4. Il résulte notamment de ces constructions que :

$$
\begin{array}{ll}
\mathrm{K} !+! \mathrm{I} !+! \mathrm{H}= & ! 7 \text { est la part harmonique de la hauteur totale de } 12 \\
\mathrm{~K} !+! \mathrm{I}= & ! 4 \text { est la part harmonique de }: \mathrm{K}+\mathrm{I}+\mathrm{H} \\
\mathrm{H}= & ! 3 \text { est la part harmonique de }: \mathrm{I}+\mathrm{H}
\end{array}
$$

Des découpes analogues, faisant intervenir le partage harmonique, se retrouvent sur des chapiteaux doriques, notamment sur ceux des Propylées d'Athènes (cf. figure 5 en annexe), mais l'indécision relative à l'unité de mesure (la valeur du pied) ne permet pas de connaître avec certitude quels étaient les nombres antiques attribués aux dimensions. Force est alors de se rabattre sur les valeurs approchées de ce partage ([4] et [17]). Il est à souhaiter que vienne le temps où les archéologues disposeront de critères objectifs pour déterminer la valeur de l'unité utilisée au lieu de se fier à des intuitions plus ou moins justifiées et qui sont loin de leur être communes à tous, ce dont témoignent les quatorze valeurs de pied proposées pour le Parthénon.

D'un point de départ trop élémentaire et au travers d'essais divers et d'erreurs redressées, nous voici, en définitive arrivés à une théorie assez cohérente. Cette théorie a l'avantage de la simplicité puisqu'elle se réduit à deux aspects complémentaires : les médiétés d'une part, et principalement les quatre médiétés de partition, les approximations d'autre part, d'ordre un ou dérivées, qui permettent d'exprimer ces médiétés en nombres entiers. Point n'aurait dû être besoin d'une dizaine d'années de tâtonnements pour ce résultat. Il suffisait de lire Vitruve, ou de savoir le lire. Certes, à l'inverse de la théorie musicale, aucun texte ni aucune tradition ne mentionnent le recours à ces médiétés dans l'architecture antique. On ne relève, et en abondance dans l'architecture romaine, que des approximations. Comme la plupart d'entre elles sont dérivées des approximations de $\sqrt{2}$ et de $\sqrt{5}$, il devait bien y avoir une raison à ce privilège pour autant, du moins, que l'on présuppose qu'un architecte ajuste les contraintes techniques et utilitaires d'un édifice à une visée esthétique qui le conduit au choix délibéré de certaines proportions.

Dans le cadre de cette théorie unitaire, tel tracé régulateur d'un monument particulier (qui en est en quelque sorte le modèle) prend un sens comme actualisation du choix d'une proportion donnée, ou de la combinaison de diverses proportions. De tels tracés, d'ailleurs, ont-ils jamais été dessinés par les architectes antiques, au moins avec la précision qu'on tient à leur donner!? Pour tracer les plans d'un temple diastyle ou eustyle, nul besoin de ces constructions géométriques précises. Il suffit d'avoir quelques notions des médiétés (tout autant qu'un musicien devait avoir quelques notions des gammes en honneur à son époque) et de disposer de tables ou d'abaques donnant les listes de couples indiquant comment couper une droite dans une proportion donnée. Il suffit pour cela d'un dessin coté, même très approximatif et sans aucune échelle, sur lequel sont reportés les nombres que donne une table.

Ces tracés ne perdent pas pour autant toute valeur à nos yeux. Tout d'abord ils nous permettent l'analyse globale d'un édifice et nous interdisent de privilégier tel 
rapport particulier isolé de son contexte. Ensuite, ils mettent en évidence la coordination des diverses dimensions d'un édifice et montrent comment leurs rapports se construisent successivement à partir d'une même grandeur de base de telle sorte que les composantes aient entre elles, comme le demande Vitruve : «!une relation précisément définie!». Enfin, ils nous permettent de disposer d'un critère de validité du modèle de coordination proposé. Ce critère est des plus contraignants puisqu'il n'admet aucun écart entre la valeur théorique proposée par le modèle et la valeur relevée sur l'édifice. Le moindre écart conduit soit à la modification du modèle, soit à son rejet pur et simple.

Ceci admis, il faut se garder d'en conclure que tout édifice antique relève de ce type d'interprétation. Vitruve tout d'abord nous préserve de cette généralisation abusive puisque, pour les cinq types de temples ioniques qu'il nous décrit, seuls en relèvent le diastyle et l'eustyle. Par ailleurs il est des monuments qui ne font intervenir aucune construction géométrique et paraissent ne jouer que sur des rapports d'entiers (cf. [36]). Il en est aussi qui font intervenir d'autres constructions tels que des triangles rectangles rationnels de type $(3,4,5)$ ou $(5,12,13)$ ou des triangles équilatéraux. Il en est enfin où l'on décèle bien le rabattement de la diagonale d'un double carré (donc $\sqrt{5}$, par exemple entre la largeur d'une baie et sa hauteur) sans qu'il y ait cependant de partage géométrique.

Pour conclure, qu'en est-il de ma problématique initiale ? Les apories de Zenon sont-elles ou non liées au problème du continu ? Les vieilles pierres ont certes parlé, mais elles ne parlent avec certitude qu'à partir de la fin du III ${ }^{\mathrm{e}}$ siècle av. J.-C. avec les grandes demeures de Pompéi. Avant la réponse reste indécise et elle le restera tant que l'on ne disposera pas d'un critère absolument indubitable pour le choix de l'unité de mesure et donc des nombres utilisés par les architectes.

Il se pourrait que la théorie des approximations soit l'un des éléments de ce critère, qui serait d'ailleurs à combiner avec d'autres relevant de l'histoire et de la géographie politique. Il est, en effet, désormais incontestable que les approximations ont été utilisées en architecture où elles ont eu la vie plus dure que les médiétés qu'elles étaient initialement chargées de traduire en «!nombres ronds!». Le problème, très vague au départ, est désormais mieux cerné et il se précise en ces termes : À quelle période ces approximations apparaissent-elles?

Revenons à l'Athénaïon de Paestum dans cette nouvelle perspective [26]. Ce temple, de la fin du VI ${ }^{\mathrm{e}}$ siècle, est plus ou moins contemporain de Zénon d'Elée. D'après les relevés de F. Krass, la hauteur de l'entablement est de 2,499, celle de l'architrave de 1,036 et celle de la frise et de ses moulures de 1,463. Le rapport de la frise à l'architrave est égal à $\frac{1,463}{2,499}=0,5854$, ce qui est la valeur approchée d'un partage harmonique, et l'anthyphérèse donne l'une des approximations d'un partage harmonique avec le couple $(41,70)$. Cependant, avec la valeur du pied proposée par Krauss, ce ne sont pas ces nombres que l'on obtient, d'où la question que je suis conduit à me poser : cette valeur est-elle la bonne ?, et ce d'autant plus que Krauss en a changé en cours d'étude (cf. figure 6 en annexe). 
Recherchant alors une valeur en accord avec les résultats de l'anthyphérèse, j'aboutis à un pied de 0,2857. Cette valeur du pied n'est pas une pure invention de ma part car elle est déjà attestée sur d'autres édifices (pied qualifié de glanique par A.!RothCongès qui en a décelé l'usage sur le site hellénistique de Glanum et qui, je suppose, a aussi été utilisé à la maison des «!Noces d'argent!» de Pompéi, pied qualifié de carolingien par F. Ulrix [38], ce qui témoignerait de sa survivance au Moyen Âge). Avec cette valeur, l'entablement aurait une hauteur de 140 dactyles, l'architrave une hauteur de 58 dactyles et la frise une hauteur de 82 dactyles. Pour les grandes dimensions, cette valeur n'est pas plus mauvaise qu'une autre. Ainsi la distance axiale des colonnes de façade est de 13,144 mètres et 46 pieds $=13,137$, la largeur du stylobate est de 14,54 mètres et 51 pieds $=14,56$; la largeur de l'architrave est de 14,14, et 49 pieds $\frac{1}{2}=14,137$, la profondeur intra-muros de la cella est de 12,286 , et 43 pieds $!=12,28$, etc.

D'autre part, les colonnes du pronaos sont de style ionique. La découpe «!naturelle!» du chapiteau ionique fait apparaître quatre hauteurs de 0,$603 ; 0,573$; 0,230 !; 0,142 . Les rapports de ces dimensions évoquent ceux d'un partage géométrique avec $\frac{0,603}{0,373}=1,616 ; \frac{0,373}{0,230}=1,621 ; \frac{0,230}{0,143}=1,619$. Or, il se trouve que, avec le pied «!glanique!» (déduit uniquement de la découpe de l'entablement) les valeurs de ces dimensions seraient assez correctement exprimées par les nombres de Fibonacci, avec 34 dactyles $=0,607(+4 \mathrm{~mm}$ sur 0,603), 21 dactyles $=0,374(+1 \mathrm{~mm}, 13$ dactyles $=$ $0,232(+2 \mathrm{~mm})$ et 8 dactyles $=0,143(+1 \mathrm{~mm})$.

Doit-on en conclure que cet architecte antique de la fin du $\mathrm{VI}^{\mathrm{e}}$ siècle a utilisé effectivement et cette valeur de pied et ces nombres ? Ce n'est pas invraisemblable, mais loin d'être assuré. Il conviendrait auparavant que d'autres acceptent ou parviennent aux mêmes résultats. Ce pourrait être le cas du Parthénon athénien du milieu du $\mathrm{V}^{\mathrm{e}}$ siècle. Parmi les quatorze valeurs de pied proposées, l'une d'entre elles, celle avancée par Jos de Waele, correspond exactement aux résultats des anthyphérèses sur les dimensions de la façade Est. À nouveau, les nombres obtenus sont ceux de la suite de Fibonacci qui conduisent à un tracé régulateur très simple et qui coordonne d'une manière très précise toutes les dimensions de cette colonnade soit à partir de la hauteur de la colonne, soit à partir de son diamètre.

À l'occasion de ces deux derniers exemples, il importe de préciser que le recours, de ma part, à l'anthyphérèse comme moyen technique de découverte de nombres simples ne présuppose aucunement que cette procédure de retranchement alterné ait été déjà inventée et utilisée à l'époque de construction de ces deux temples. Il existe, en effet, d'autres moyens plus rudimentaires pour trouver ces couples de nombres, moyens que l'on pourrait même qualifier d'empiriques. Il suffit, par exemple, d'écrire la liste des entiers, celle de leurs carrés et celle du double de ces carrés puis de rechercher sur les deux dernières listes deux nombres qui ne diffèrent que d'une unité. La procédure élaborée peut donc n'être pas encore inventée mais la présence de ces couples de nombres qui approximent $\sqrt{2}, \sqrt{5}$ ou $\varnothing$ n'en témoigne pas moins et de la conscience 
de la difficulté que posent les grandeurs non commensurables et du désir de les exprimer pas des entiers de la manière la plus exacte possible.

On ne relève, à ma connaissance, d'approximations que chez les Grecs. Les babyloniens, comme en témoigne une tablette datée de la première dynastie (entre -1800 et -1500), calculaient, et de manière très précise, des valeurs approchées par des procédures conduisant à l'extraction de racines carrées [15]. Les Grecs ont développé un autre mode d'approche par des couples d'entiers. On peut évidemment se demander quelle en est raison et si ce ne serait pas sur une demande extérieure : musiciens ayant besoin de connaître la longueur de deux cordes pour réaliser quinte, quarte ou couper un octave, architectes aussi pour inscrire une porte dans un rectangle harmonique ? En l'absence de textes, toutes les hypothèses sont permises et elles doivent donc rester de simples hypothèses.

Le type de démarche adoptée ici pour l'étude d'un monument risque de surprendre les archéologues ou les architectes spécialistes de l'Antiquité peu accoutumés, du fait qu'ils ne sont nulle part enseignés, aux concepts sur lesquels repose la théorie proposée. L'historien de la mathématique ancienne ne se préoccupe ni d'implantation, ni des techniques de construction, ni du programme iconographique, ni des éléments d'une modénature $^{8}$. Par contre, en l'absence de textes, il cherche des indices, non pas de la mathématique pure, mais de ses retombées, de ses utilisations. Toute collection de mesures relevées sur un édifice est, pour lui, témoignage potentiel d'inter-relations ordonnées par un principe d'origine mathématique qu'il serait heureux de découvrir et il lui arrive parfois de regretter de ne pas disposer de toutes les mesures qui lui seraient utiles. Il lui semble alors que l'archéologue fait preuve de méfiance envers ce qui n'est que numérique. Sans doute est-ce simplement parce qu'un archéologue sérieux ne saurait en donner une interprétation rationnellement satisfaisante et qui échapperait aux pièges de la numérologie et de la mystique des nombres. La théorie proposée échappe, me semble-t-il, à ces excès. Ni archéologue ni architecte, ni strict historien des sciences, par hasard et par chance à la frontière de deux disciplines, je m'efforce simplement de transposer en l'une les apports de l'autre afin de mieux deviner la genèse du plan d'un temple et de mieux reconstituer les premières ébauches d'une théorie mathématique.

Bibèmus, juillet 1990

\section{BIBLIOGRAPHIE}

[1] AMY R. et al., L'arc d'Orange, 15e suplément de Gallia, CNRS, Paris, 1962.

[2] AMY R. et GROS P., La Maison Carrée de NIMES, $38^{\mathrm{e}}$ supplément de Gallia, CNRS, Paris, 1979.

\footnotetext{
${ }^{8}$ Modénature!: profil de monture (N.D.L.R.).
} 
[3] BALANOS N., Les monuments de l'Acropole, relèvement et restauration, Charles Massin, Paris, 1938.

[4] BOHN. R., Die Propylaen der Akropolis zu Athen, Berlin/Stuttgart, 1882.

[5] CLEYET-MICHAUD M., Le nombre d' or, Que sais-je ?, nº 1530, P.U.F., 1978.

[6] COULTON J.-J., Greek architects at work, Problems of structure and design, Granada, USA, 1982.

[7] COULTON J.-J., «!Modules and Measurements in Ancient Design and Modern Scolarship!», Munus non ingratum, Actes du colloque de Leyde de 1987, ed. Geertman and De Jong, Leiden, 1989, p. 85-89.

[8] DE L'ORME P., De l'Architecture, réédition de 1648, P. Madarga, Bruxelles, 1981.

[9] DE WAELE J., La progettatzione dei templi dorici di Himera, Segesta e Siracusa, Secondo quaterno Imerese «!L'Herma!», Roma, 1982.

[10] DE WAELE J., Der entwurf des Héraïon von Olympia, Babesh, n 57, 1982.

[11] DE WAELE J., Mnesikles' design of the Propylaia in Athens, [à paraître].

[12] DHOMBRES J., Nombre, Mesure et Continu, Epistémologie et Histoire, Publication de l'I.R.EM. de Nantes, CEDIC - F. Nathan, Paris, 1978.

[13] FOWLER D., Ratio in early greek mathematics, Bul. of Amer. Math. Soc. 1, 1979 , p. 807-846.

[14] FOWLER D., The mathematics of Plato's academy : a new reconstruction, Clarendon Press, 1987.

[15] FREY L., «!À propos d'approximations!», L’à-peu-près, Aspects anciens et modernes de l'approximation, E.H.E.S.S., Paris, 1988, p. 11-25.

[16] FREY L., «!Besançon : La Porte noire - Carrés et diagonales!», Mathématiques, Informatique et Sciences Humaines, $\mathrm{n}^{\circ} 105,1989$, p. 27-62.

[17] FREY L., «!Données architecturales et hypothèses sur la mathématique préeuclidienne : le partage harmonique!», Munus non ingratum, Actes du colloque de Leyde de 1987, ed. Geertman and De Jong, Leiden, 1989, p. 90-99.

[18] FREY L., «!Médiétés et approximations chez Vitruve!», Revue Archéologique, fascicule 2, 1990.

[18bis] FREY L., «!Le trésor de Thèbes!: deux modèles pour un monument!», Mathématiques et Sciences humaines, $\mathrm{n}^{\circ}$ 87, 1984, p. 33-66.

[19] GROS P., Vitruve - livre III, Collection des Universités de France, Paris, 1990.

[20] GROS P., Nombres irrationnels et nombres parfaits chez Vitruve, M.E.F.R.A., tome 88, 1976, 2, p. 669-704.

[21] GUILBAUD G.-Th., Leçons d' à peu près , Paris, Christian Bourgeois, 1985.

[22] HEATH T., A history of greek mathematics, Oxford, Clarendon Press, 2 tomes, 1921-1960. 
[23] HEATH T., Diophantus of Alexandria, A Study in the History of Greek Algebra, New York, Dover books, 1885-1910-1964.

[24] ITARD J., Les livres arithmétiques d'Euclide, Paris, Hermann, 1961.

[25] IVOR T., Greek Mathematical Works, Textes et traduction anglaise, 2 tomes, Loeb Classical Library, n 335, 336, H.U.P., Cambridge, 1967.

[26] KRAUSS F., Die tempel von Paestum, 1 : Des Athena tempel, De Gruyter, 1959.

[27] MERTENS D., Der tempel von Segesta, Verlag Ph. von Zabern, Mainz am Rhein, 1984.

[28] MICHAUD J.-P., Le trésor de Thèbes, Publications de l'École française d'Athènes, Paris, de Boccard, 1973.

[29] MICHEL P.-H., De Pythagore à Euclide, Contribution à l'histoire des mathématiques pré-euclidiennes, Paris, Les Belles Lettres, 1957.

[30] NICOMAQUE de Gerase, Introduction Arithmétique, Traduction Janine BERTIER, Paris, Vrin, 1978.

[31] PAPPUS d'Alexandrie, La collection mathématique, Traduction P. Ver EECKE, Paris, Blanchard, 1982.

[32] PETERSE C.L.J., Note on the design of the house of Pansa (VI,6,1) in Pompéi, Overdruck uit Mededelingen Rome, NS 11 - deel XLVI.

[33] SARRADE M -Th., Sur les connaissances mathématiques des bâtisseurs de cathédrales, Paris, Librairie du Compagnonage, 1986.

[34] SOUBIRAN, Vitruve - Livre IX, Collection des Universités de France, Paris, 1969.

[35] TANNERY P., Du rôle de la musique grecque dans le développement de la mathématique, Mémoires scientifiques, 71, 1902, in : Bibliotheca mathematica, 3, Folge, t. III, 1902, pp 161-175.

[36] THEODORESU D., «!Le forum et le temple «!dorique-corinthien!» de Paestum : une expérience pré-vitruvienne!», Munus non ingratum, Actes du colloque de Leyde de 1987, ed. Geertman and De Jong, Leiden, 1989, p. 114-115.

[37] THEON de Smyrne, Des Connaissances Mathématiques Utiles pour la Lecture de Platon, Texte et traduction J. Dupuis, Paris, 1892.

[38] ULRIX F., Propos sur la métrologie du Moyen Âge en architecture, Annales de la Fédération archéologique et historique de Belgique, 40e session, 7, p. 683-696.

[39] WALter H., La Porte Noire de Besançon, Contribution à l'étude de l'art triomphal des Gaules, avec relevés de J. Bruchet, Paris, Belles!Lettres, 1985.

[40] WARUSFELD A., Les Nombres et leurs Mystères, Paris, Le Seuil, 1961.

En outre, les membres du bureau d'Aix de l'Institut de Recherche sur l'Architecture Antique (C.N.R.S.) ont eu l'amabilité de me faire part de leurs travaux et de leurs résultats avant publication, notamment : 
FINCKER M. pour les amphithéâtres d'Arles et de Nîmes et les temples de Belo.

PAILLET J.-L. pour le macellum et la basilique de Belo et pour l'aqueduc de Nîmes.

ROTH-CONGES A., pour divers édifices de Glanum

VARENE P., $\quad$ pour l'enceinte de Nîmes. 


\section{ANNEXES}

\section{Constructions géométriques}

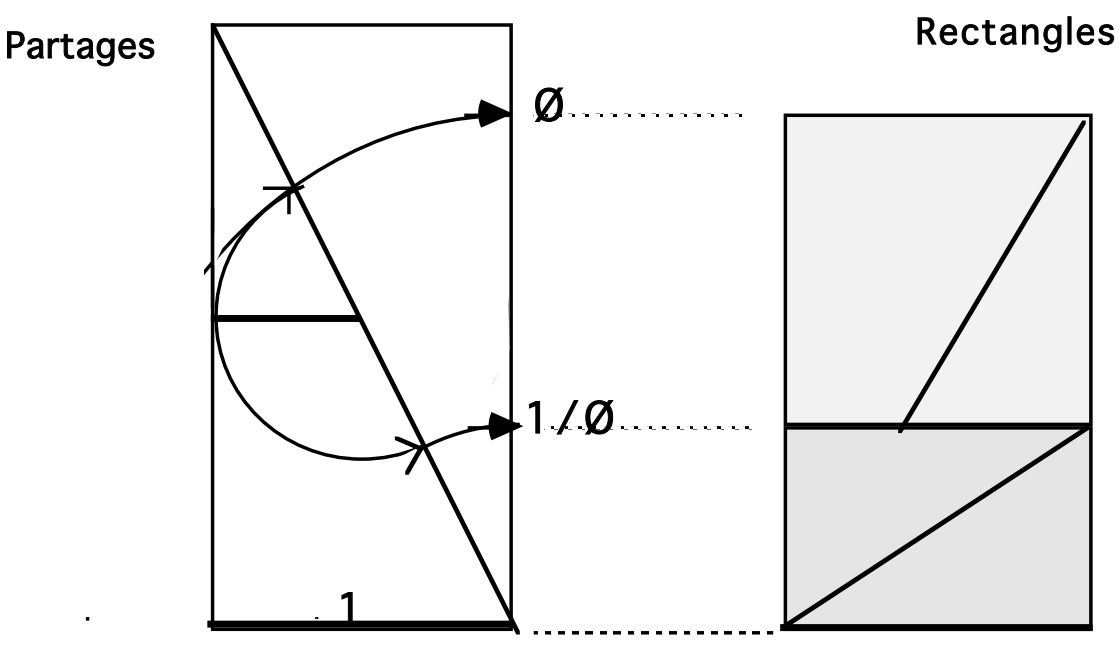

Figure 1 : Partage géométrique d'une droite $-\varnothing=(\sqrt{ } 5=1) / 2$

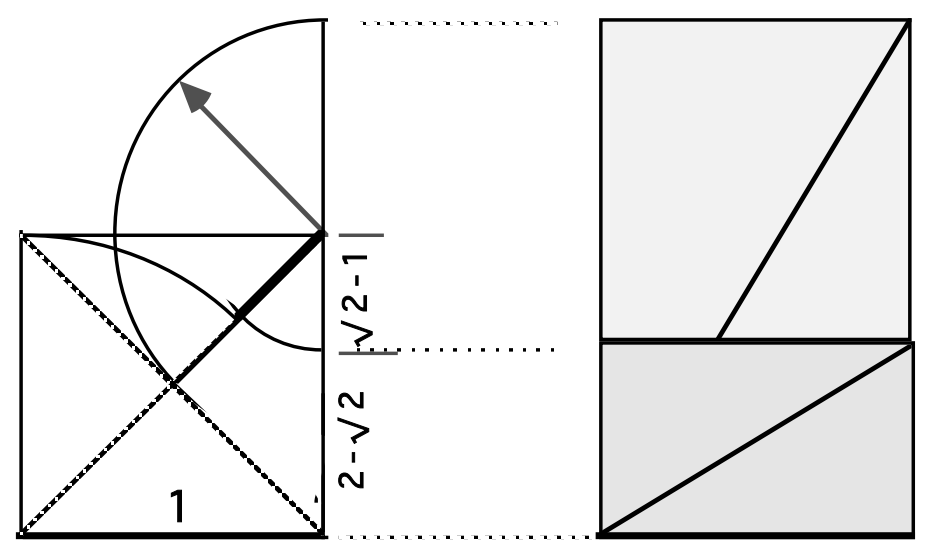

Figure 2 : Partage harmonique d'une droite

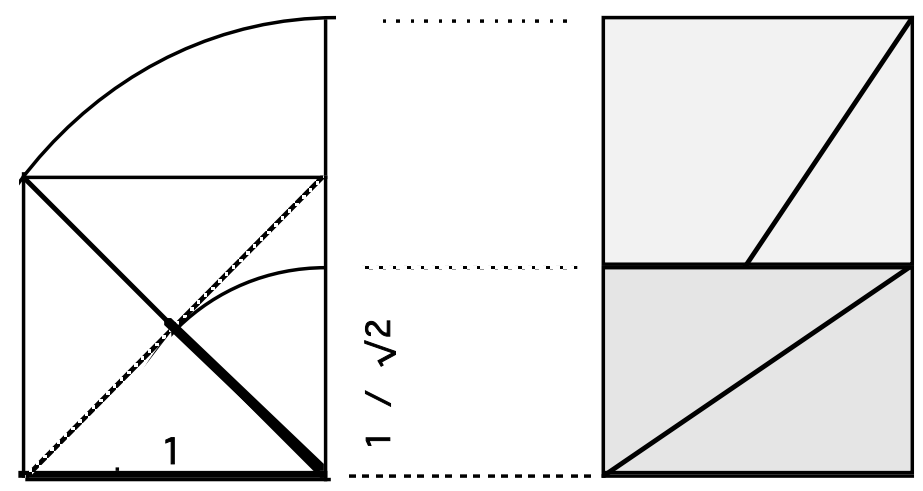

Figure 3 : Partage sous-harmonique d'une droite 


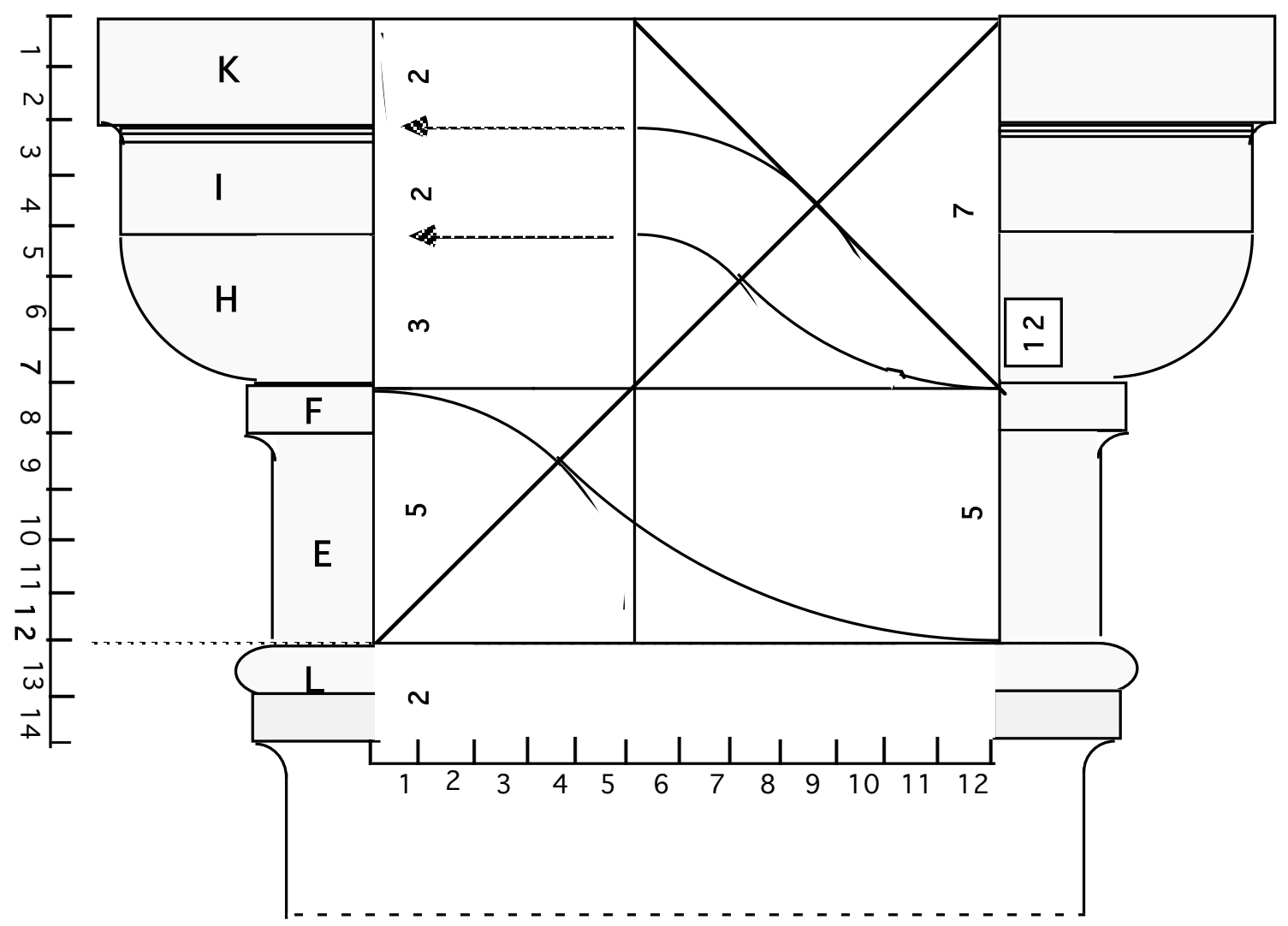

Figure 4 : Philibert de l'Orme : la découpe du chapiteau toscan
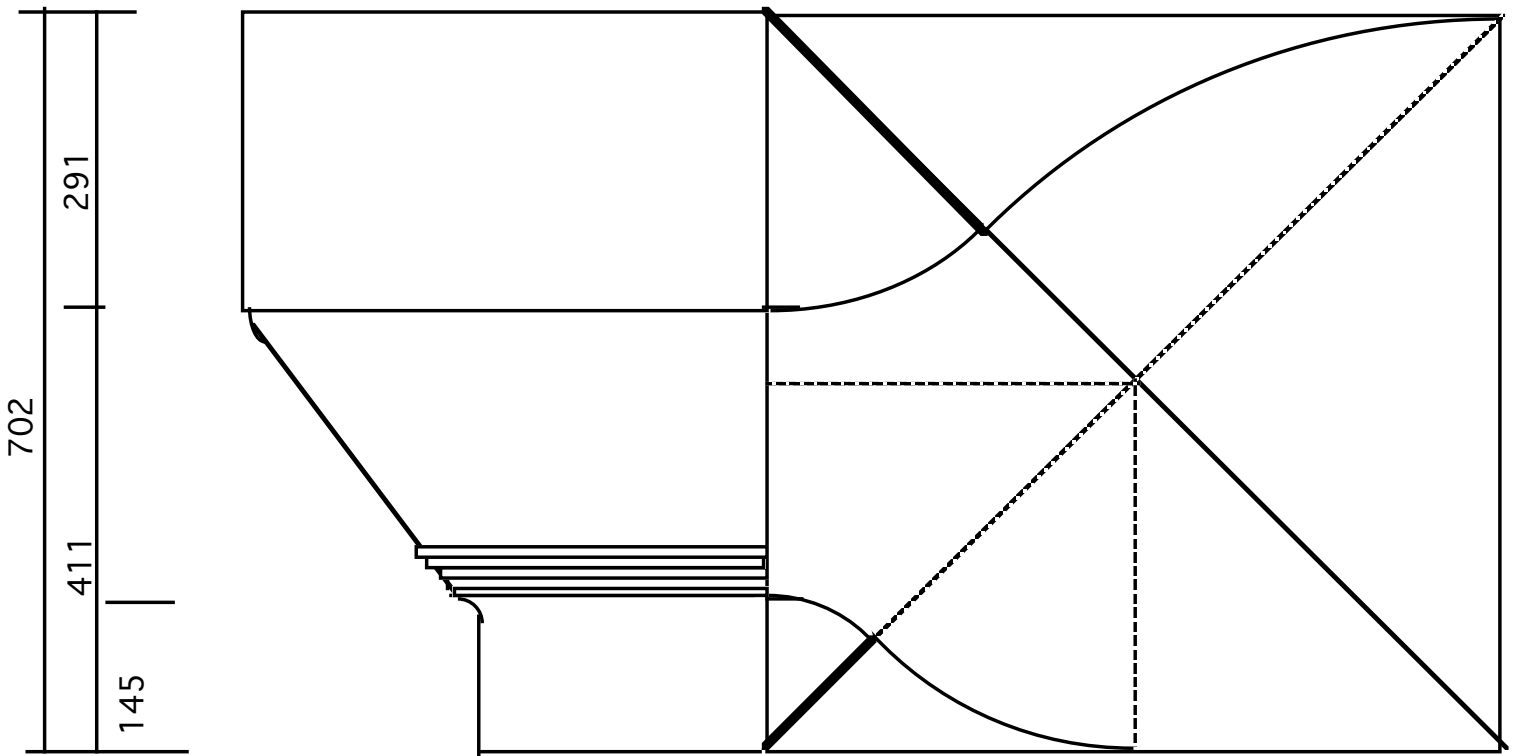

Figure 5: ATHENES, Les Propylées - Découpe du chapteau dorique 


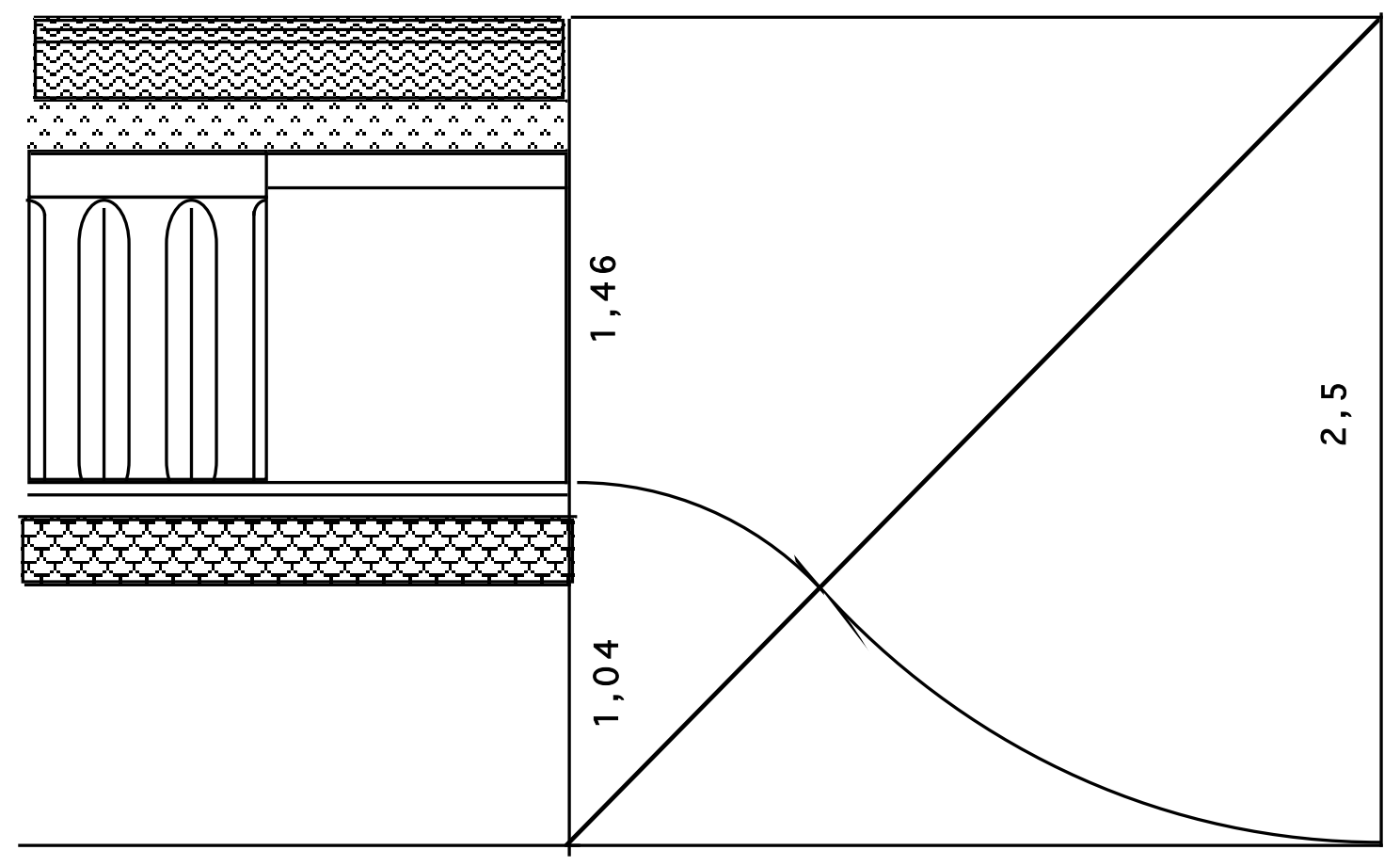

Figure 6 : Paestum - Athénaïon : Partages de la hauteur de l'entablement 